\title{
Effect of Different Softeners on Dimensional Stability and Color Fastness Properties of Stretch Denim Fabric
}

\author{
Faruq Hosen, Md. Zayedul Hasan, A. K. M. Ayatullah Hosne Asif* \\ Department of Textile Engineering, Mawlana Bhashani Science and Technology University, Santosh, Tangail, Bangladesh
}

Email address:

ayatasif.mbstu@gmail.com (A. K. M. A. H. Asif)

${ }^{*}$ Corresponding author

\section{To cite this article:}

Faruq Hosen, Md. Zayedul Hasan, A. K. M. Ayatullah Hosne Asif. Effect of Different Softeners on Dimensional Stability and Color Fastness Properties of Stretch Denim Fabric. Advances in Applied Sciences. Vol. 5, No. 4, 2020, pp. 112-119. doi: 10.11648/j.aas.20200504.13

Received: October 16, 2020; Accepted: October 28, 2020; Published: November 11, 2020

\begin{abstract}
This study was designed to investigate the effect of different softeners on dimensional stability and color fastness properties of stretch denim fabric. Stretch denim fabric was washed using enzyme concentration of $4.0 \mathrm{~g} / \mathrm{l}$ for 30 minutes in $40^{\circ} \mathrm{C}$ temperature at $\mathrm{pH} 5.5$ and then different softeners were applied in conjunction with standard recipes. Different color fastness properties, dimensional stability were analyzed in combination with enzyme wash and stone enzyme wash along with cationic, anionic, nonionic, micro silicone, macro silicone and nano silicone softeners. Stone enzyme wash treated with different softeners on stretch denim fabric demonstrated better performance in case of different color fastness properties rather than enzyme wash. No significant change was observed for application of different kinds of softeners on enzyme wash and stone enzyme treated stretch denim fabric regarding the grade of color change and color staining for color fastness to light and color fastness to water. Silicone-based softeners illustrated better performance for color fastness to washing than other softeners for both enzyme wash and stone enzyme wash. Cationic softener proven excellent durability and suitability towards multiple washes rather than other softeners. The GSM of stretch denim fabric also increased accordingly after applying different softeners rather than untreated stretch denim fabric. From scanning electron microscope images, stretch denim fabric sample treated with enzyme and cationic, micro silicone softeners implied less ruptures and less cracks rather than stone enzyme treated with cationic, micro silicone softeners.
\end{abstract}

Keywords: Stretch Denim, Washing, Softener, Color Fastness, Shrinkage

\section{Introduction}

Denim is strong, stiff, hard-wearing woven fabric. Denim is one of the oldest fabrics in the world most commonly associated with jeans [1]. Stretch denim is a new type of fabric (cotton / polyester blend) which integrates a small amount of elastane, stretch, synthetic fiber, also known as spandex, into the fabric. Stretch jeans typically include about one to three percent elastane [2]. Denim is made of a twill weave with indigo dyed warp and white weft yarns. The white yarns run across the width of the fabric (the weft). In the modern textile industry, enzymes are applied mainly to get a cleaner fabric surface [3]. They played an imperative role not only to provide an aesthetic finish but also enhanced the appeal in addition to reduce fuzz and pills, increase smoothness, softness, luster and brightness, improve fabric handle and drape ability and increase the vintage effects which meet the requirement of modern denim fashion trend [4-6].

Washing is such kind of technology which is used to modify the appearance, size, outlook, comfortability and fashion of the garments [7, 8]. Garment becomes lucrative, efficient and glossy appearance by washing process [9]. Washing operations are carried out most frequently during a complete textile finishing cycle [10]. Washing is a process which is connected to key treatment and aimed at removing insoluble matters or other impurities from the fabric [11]. In denim garment, washing is also performed to produce effects such as color fading with or without patchiness, crinkles, puckering, hairiness, softened hand feel, and stabilize dimensions [12]. Various research works encompassed about the evaluation of the characteristics of acid wash, nano mineral washing and finishing with different chemicals for denim fabric along with comparing the luxury features among the different types of finishing chemicals used to treat 
and analysis the surface modification, color fading of denim fabrics $[13,14]$.

In classical denim washing, softening is the last step before the drying process $[15,16]$. To improve fabric handle and other valuable properties, softeners are widely used in finishing operations $[17,18]$. Although enzymatic method is eco-friendly but enzyme treatment on cellulosic garment degrades cellulose chains, yielding shorter chain in cellulose polymers [19]. Washing of denim garments is one of the most essential processes [20]. This process adds a lot of value to the final garment [21]. In the washing process, there are various methods to impart faded effects. The traditional washing is done by using stone washing, washing with strong bleaching agents such as sodium hypochlorite, potassium permanganate; however, these processes are not environment friendly $[22,23]$. Alternative methods have been developed where cellulase enzymes are used in place of pumice stone to impart abraded effect [24]. Recent development of different types of denim which are soft, light and comfortable in all seasons, have added a new dimension to the fashion world. The major aim of the research was to study the effect of different softeners on dimensional stability and color fastness properties of stretch denim fabric treated with enzyme and stone enzyme wash.

\section{Materials and Methods}

\subsection{Material}

Fabric:

In this research work, $98 \%$ of cotton $2 \%$ of elastane indigo dyed stretch denim fabrics was used. The fabric GSM (grams per square meter) was 330 (Before wash), 3/1 right hand twill fabric with width 57 inch.

Chemicals:

For experimentation, desizing agent soda ash (sodium carbonate) was used. This chemical was imported from China. Detergent (Suntex, HF) was imported from Germany. Enzyme (HIDROS TED, neutral enzyme-liquid form), different softeners like cationic, anionic, nonionic, silicone (micro, macro, nano) softeners were also used for this study.

Instruments:

For this experiment, washing machine, hydro extractor machine, tumble dryer machine, fabric GSM cutter, electronic balance and fabric $\mathrm{pH}$ meter were used.

\subsection{Methodology}

\subsubsection{Working Procedure of Enzyme Wash}

(i) Desizing

In the garments washing, desizing is the first step of enzyme wash. This step was used for removing dirt, dust and other foreign materials from garments surface. Here wetting agent was used to increase the penetration property of liquid into the garments. Material and liquor ratio was kept at 1:8; then started the machine by adding de-sizing agent $4.0 \mathrm{~g} / 1$, soda ash $2.0 \mathrm{~g} / \mathrm{l}$ and wetting agent $1.5 \mathrm{~g} / \mathrm{l}$. The temperature was kept $60^{\circ} \mathrm{C}$ for 20 minutes. Then the liquor was dropped out by following cold wash.

(ii) Bio-abrasion

In this step hairy fibers and coloring materials were removed by the activity of enzyme. If more fading is required then stone has to be used at half the volume of garments. Material and liquor ratio were kept at 1:8. Then machine was run by adding anti back staining agent at about $2.0 \mathrm{~g} / 1$ with $4.0 \mathrm{~g} / 1$ neutral enzyme-liquid form. The calculated $\mathrm{pH}$ for enzyme was 5.5 , and the temperature was kept $40^{\circ} \mathrm{C}$ for 30 minutes. Then dropped the liquor and cold wash was done.

(iii) Back Wash

This step was done to resist the activity of enzyme. In this step all the enzyme be dead due to high temperature. Then dead enzymes and coloring material and other impurities were removed from the garments by dropping the liquor and dye again a rise wash by material and liquor ratio of $1: 6$; then ran the machine by adding $3.0 \mathrm{~g} / \mathrm{l}$ anti back staining with addition of soda ash $4.0 \mathrm{~g} / 1$. The temperature was kept $50^{\circ} \mathrm{C}$ to run for 5 minutes. Then dropped the liquor and rinsing was done.

\section{(iv) Softener Wash}

Subsequently cationic, anionic, nonionic, micro, macro and nano silicone softener was applied on enzyme washed stretch denim fabric and the temperature was kept cold to run for 5 minutes. Then liquor was dropped by following rinse wash.

(v) Hydro Extracting

After the above washing process, the liquor was dropped followed by unloading the garments and hydro extracted at 200 rpm for 3-4 min to remove the excess water. At last sample was dried at $75^{\circ} \mathrm{C}$ for $35-40 \mathrm{~min}$ by steam dryer.

\subsubsection{Working Procedure of Stone Enzyme Wash}

Stone washing process used to produce worn, dilapidated appearance on denim fabric. This stone washing process was carried out by washing stretch denim fabric with pumice stones in a rotating drum by using chemicals to create desired appearance. In this experiment, stone enzyme washing process was done by different steps like desizing, bioabrasion, adding and tumble of pumice stones with enzyme along with acetic acid and anti-stain agent at $40-50^{\circ} \mathrm{C}$ for 40 60 mins depending on the desired outlook, drain the liquor and separate the garments from pumice stones, softening with cationic, anionic, nonionic, silicone-based softeners accordingly and hydro extracting procedures.

\subsubsection{Scanning Electron Microscopy}

Scanning electron microscopy (SEM) (JSM-6490 LA, JEOL, Tokyo, Japan) was used to understand the surface morphology of the enzyme treated and enzyme stone treated samples. The test was conducted using the equipment at the University of Dhaka (Center for Advanced Research in Sciences) with a maximum operating range of $20 \mathrm{kV}$. By scanning the sample surface with a high energy beam of electrons, the scanning electron microscope took an image. The electron associate with the atoms in the sample, triggering signals which provide information about the surface topography of the sample. 


\subsubsection{Color Fastness Measurement}

The following color fastness tests were done for conducting this research work [25-28]:

1) Color fastness to wash (Method: ISO $105 \mathrm{C} 10)$

2) Color fastness to rubbing (Method: ISO $105 \mathrm{X12}$ )

3) Color fastness to light (Method: ISO 105 B02)

4) Color fastness to water (Method: ISO 105 E01)

\section{Results and Discussion}

\subsection{Effect of Different Types of Softeners on Dimensional Stability of Stretch Denim Fabric}

i) For Enzyme Wash

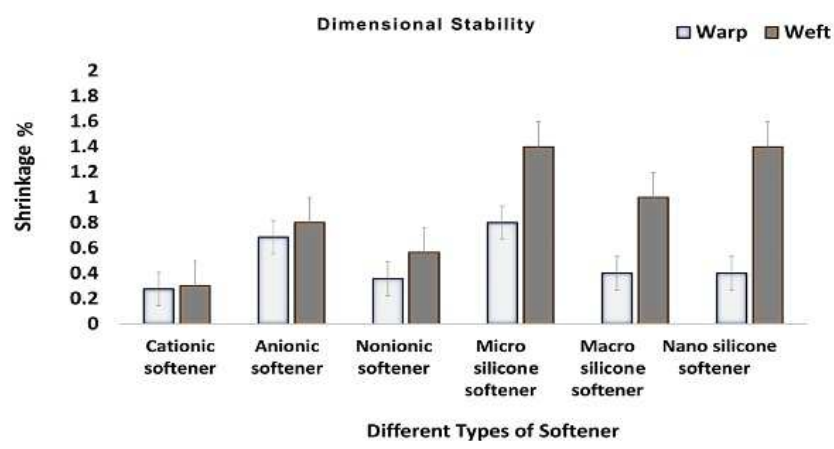

Figure 1. Variation of softeners on dimensional stability of enzyme treated stretch denim fabric.

Following figure demonstrates about the dimensional stability of enzyme washed sample treated with cationic, anionic, nonionic softener and silicone-based softeners for stretch denim fabric. Dimensional stability of enzyme washed sample with cationic, nonionic softeners were better than the other softeners. Enzyme wash with cationic softener showed prodigious result compared to others because when this type of softener was added to the process it controlled the dimensional stability. To check the changes in dimensional stability, the shrinkage (\%) were measured [29]. Cationic softener was more durable and pertinent to multiple washes rather than other softeners.

ii) For Stone Enzyme Wash

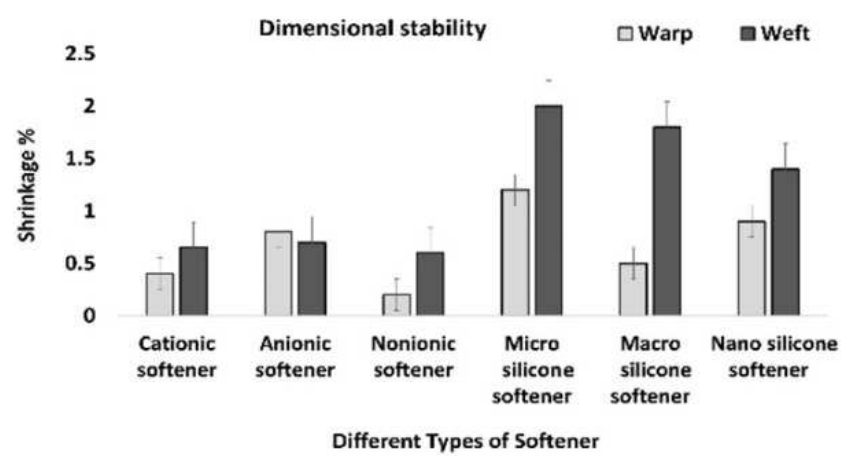

Figure 2. Variation of softeners on dimensional stability of stone enzyme treated stretch denim fabric.

Following figure shows about the dimensional stability of enzyme stone washed sample treated with cationic, anionic, nonionic softener and silicone-based softeners for stretch denim fabric. Dimensional stability of enzyme stone washed sample with cationic, anionic, nonionic softener was better than the silicone-based softeners. Among them, the nonionic softener treated sample exhibited the best result in terms of dimensional stability because it withstands the repercussion of hard water, acid or base environment and also stable in presence of cations and anions. Stone enzyme wash with cationic softener also showed acceptable result.

\subsection{Effect of Different Softeners on Fabric GSM of Stretch Denim Fabric}

\section{i) For Enzyme Wash}

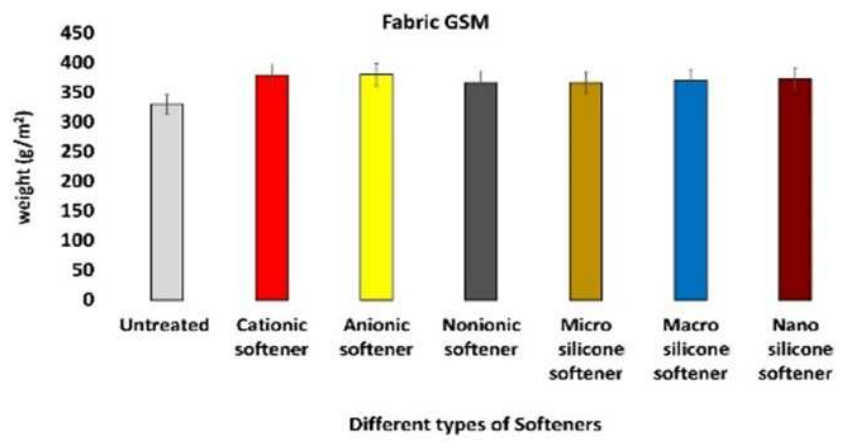

Figure 3. Variation of softeners on fabric GSM for enzyme treated stretch denim fabric.

The bar diagram represents about the effect of different softeners on fabric GSM of enzyme treated stretch denim fabric. It was observed that the maximum fabric weight was achieved on cationic and anionic softener treated sample due to impart antistatic properties from the enzyme wash process. Cationic softener was considered as the best softeners and that was applied by exhaustion to all fibers from a high liquor to goods ratio as they provide a hydrophobic surface. The hydrophobic groups were oriented away from the fiber surface. On the other hand, when different types of softeners were applied on stretch denim fabric, then the weight of fabric was increased accordingly for different types of softeners.

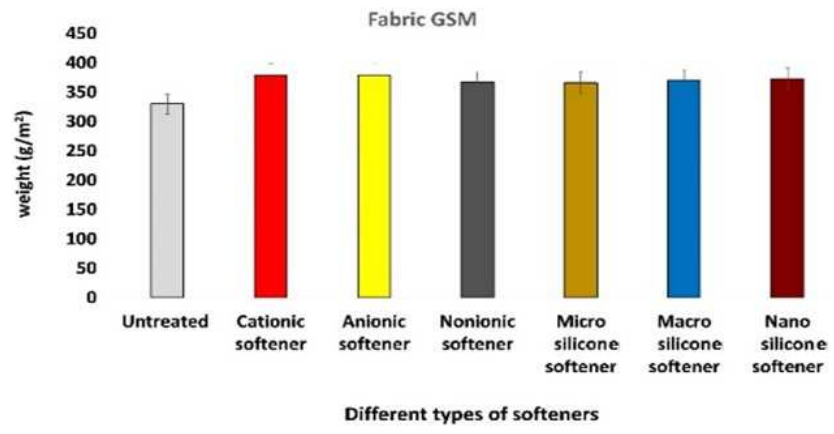

Figure 4. Variation of softeners on fabric GSM for stone enzyme treated stretch denim fabric.

ii) For Stone Enzyme Wash

Above figure represents about the effect of softeners on fabric GSM of stone enzyme treated stretch denim fabric. It 
was observed that the maximum fabric weight was achieved for cationic and anionic softener treated sample due to their strong antistatic effects and good rewetting properties. Cationic and anionic softeners contain lubricating properties which increased the fabric weight in both cases during enzyme wash and stone enzyme wash. In stone washing, the friction between stones and fabric took place because of using pumice stones.

\subsection{Effect of Different Softeners on Color Fastness to Wash for Enzyme Treated Stretch Denim Fabric}

Table 1. Color fastness to wash for enzyme treated stretch denim fabric.

\begin{tabular}{|c|c|c|c|c|c|c|c|c|}
\hline \multirow{2}{*}{ Sl. No. } & \multirow{2}{*}{ Different Types of Softener } & \multirow{2}{*}{$\begin{array}{l}\text { Grade (Change in } \\
\text { Color) }\end{array}$} & \multicolumn{6}{|c|}{ Grade (Color Staining on Multi Fiber) } \\
\hline & & & Acetate & Cotton & Nylon & Polyester & Acrylic & Wool \\
\hline 1 & Cationic Softener & 3 & 3 & $3-4$ & 3 & $3-4$ & 3 & $3-4$ \\
\hline 2 & Anionic Softener & 4 & 4 & $4-5$ & $3-4$ & 4 & $4-5$ & $4-5$ \\
\hline 3 & Nonionic Softener & 4 & 4 & 4 & 4 & 4 & 4 & 4 \\
\hline 4 & Micro Silicone Softener & $4-5$ & 4 & 4 & $3-4$ & $4-5$ & 4 & $4-5$ \\
\hline 5 & Macro Silicone Softener & 4 & 4 & 4 & 4 & $4-5$ & 4 & 4 \\
\hline 6 & Nano Silicone Softener & $4-5$ & 4 & 4 & 4 & $4-5$ & $4-5$ & $4-5$ \\
\hline
\end{tabular}

Following table demonstrates about color fastness to wash of enzyme treated sample with cationic, anionic, nonionic softener and silicone-based softeners. It was observed that color change and color staining result of cationic softener was comparatively poor than the other softeners. In case of only enzyme washed sample with cationic softener, the percentage of unfixed dyes were higher. Color fastness to wash for anionic, nonionic and silicone-based softeners were better than that of cationic softener. The nature of cationic softener changed dyeing shade, so grading of color change and color staining for color fastness to wash was decreased.

\subsection{Effect of Different Types of Softeners on Color Fastness to Wash for Stone Enzyme Treated Stretch Denim Fabric}

Table 2. Color fastness to wash for stone enzyme treated stretch denim fabric.

\begin{tabular}{|c|c|c|c|c|c|c|c|c|}
\hline \multirow{2}{*}{ SI. No. } & \multirow{2}{*}{ Different Types of Softener } & \multirow{2}{*}{$\begin{array}{l}\text { Grade (Change } \\
\text { in Color) }\end{array}$} & \multicolumn{6}{|c|}{ Grade (Color Staining on Multi Fiber) } \\
\hline & & & Acetate & Cotton & Nylon & Polyester & Acrylic & Wool \\
\hline 1 & Cationic Softener & 4 & 4 & $3-4$ & 4 & 4 & $3-4$ & 4 \\
\hline 2 & Anionic Softener & 4 & $4-5$ & $4-5$ & $4-5$ & 4 & $4-5$ & $4-5$ \\
\hline 3 & Nonionic Softener & 4 & 4 & 4 & 4 & $4-5$ & 4 & 4 \\
\hline 4 & Micro Silicone Softener & $4-5$ & $4-5$ & $4-5$ & $4-5$ & $4-5$ & 4 & $4-5$ \\
\hline 5 & Macro Silicone Softener & 4 & $4-5$ & 4 & 4 & 4 & 4 & 4 \\
\hline 6 & Nano Silicone Softener & $4-5$ & 4 & $4-5$ & $4-5$ & 4 & $4-5$ & $4-5$ \\
\hline
\end{tabular}

The following table represents about the effect of different types of softeners on color fastness to wash for stone enzyme treated stretch denim fabric. It was observed that color change and color staining result for enzyme stone wash was relatively good. For stone enzyme washed sample the percentage of unfixed dyes were lower which caused the better result in color change and color staining. Color fastness to wash for different silicone-based softeners represented better performance rather than others. This was due to the fact that silicones are macromolecules containing a connector of contrasting atoms of silicon and oxygen with silicon-attached organic groups. The softening capability of silicone comes from the versatility of the siloxane backbone and its flexibility of motion along the Si-O bonds. Silicones are macromolecules consisting of a polymer backbone of silicone and oxygen alternating atoms, of organic silicone groups. The power to relieve silicone comes from the flexibility and the freedom of rotation of the siloxane backbone along Si-O bonds. So, they imparted a decent feature about color fastness to wash. They made a lubrication layer on the surface which was relatively waterproof and offered fabrics a silky, smooth touch. When fabric was treated with stone enzyme wash along with cationic softener, the usual poor performance of cationic softener in case of color fastness to wash became little bit improved as the stone wash removed the unfixed dyes considerably.

\subsection{Effect of Different Types of Softeners on Color Fastness to Rubbing for Enzyme Treated Stretch Denim Fabric}

Table 3. Color fastness to rubbing for enzyme treated stretch denim fabric.

\begin{tabular}{llcc}
\hline Sl. No. & Different Types of Softener & $\begin{array}{l}\text { Grade (Dry } \\
\text { rubbing) }\end{array}$ & $\begin{array}{c}\text { Grade (Wet } \\
\text { rubbing) }\end{array}$ \\
\hline 1 & Cationic Softener & $2-3$ & 2 \\
2 & Anionic Softener & 3 & $2-3$ \\
3 & Nonionic Softener & $2-3$ & 2 \\
4 & Micro Silicone Softener & 3 & 2 \\
5 & Macro Silicone Softener & 3 & $2-3$ \\
6 & Nano Silicone Softener & 3 & $2-3$ \\
\hline
\end{tabular}

Succeeding table characterizes the effect of different softeners on color fastness to rubbing of enzyme treated stretch denim fabric. It was observed that enzyme washed samples; tested for wet and dry rubbing result was comparatively poor. The reason behind that, in case of only enzyme washed sample, the percentage of unfixed dyes was higher which caused for poor wet rubbing results. Enzyme 
wash with cationic, anionic, nonionic and silicone-based softeners signified relatively same grade for color fastness to dry and wet rubbing.

\subsection{Effect of Different Types of Softeners on Color Fastness to Rubbing of Stone Enzyme Treated Stretch Denim Fabric}

Table 4. Color fastness to rubbing of stone enzyme treated stretch denim fabric.

\begin{tabular}{llcc}
\hline SI. No. & Different Types of Softener & $\begin{array}{l}\text { Grade (Dry } \\
\text { rubbing) }\end{array}$ & $\begin{array}{c}\text { Grade (Wet } \\
\text { rubbing) }\end{array}$ \\
\hline 1 & Cationic Softener & 3 & 2 \\
2 & Anionic Softener & 3 & $2-3$ \\
3 & Nonionic Softener & $3-4$ & 3 \\
4 & Micro Silicone Softener & 4 & $3-4$ \\
5 & Macro Silicone Softener & 4 & $3-4$ \\
6 & Nano Silicone Softener & 4 & $3-4$ \\
\hline
\end{tabular}

Following table epitomizes the effect of different softeners on color fastness to rubbing of enzyme stone treated stretch denim fabric. It was observed that enzyme stone washed sample was tested for wet and dry rubbing result was comparatively good than enzyme wash sample. The reason behind that in case of only enzyme washed sample, the percentage of unfixed dyes was higher which caused poor wet rubbing results. Enzyme wash with cationic, anionic, nonionic softener and silicone-based softeners treated fabric was relatively same grade for color fastness to dry and wet rubbing. Besides, stone enzyme wash with different siliconebased softeners represented better grading rather than cationic, anionic and nonionic softeners for color fastness to dry and wet rubbing.

\subsection{Effect of Different Types of Softeners on Color Fastness to Water for Enzyme Treated Stretch Denim Fabric}

Table 5. Color fastness to water for enzyme treated stretch denim fabric.

\begin{tabular}{|c|c|c|c|c|c|c|c|c|}
\hline \multirow{2}{*}{ Sl. No. } & \multirow{2}{*}{ Different types of Softener } & \multirow{2}{*}{$\begin{array}{l}\text { Grade (Change } \\
\text { in Color) }\end{array}$} & \multicolumn{6}{|c|}{ Grade (Color Staining on Multi Fiber) } \\
\hline & & & Acetate & Cotton & Nylon & Polyester & Acrylic & Wool \\
\hline 1 & Cationic Softener & 4 & 4 & 4 & 4 & $4-5$ & 4 & 4 \\
\hline 2 & Anionic Softener & $4-5$ & 4 & $4-5$ & $4-5$ & 4 & $4-5$ & $4-5$ \\
\hline 3 & Nonionic Softener & 4 & $4-5$ & $4-5$ & 4 & 4 & 4 & 4 \\
\hline 4 & Micro Silicone Softener & $4-5$ & 4 & 4 & $4-5$ & $4-5$ & 4 & $4-5$ \\
\hline 5 & Macro Silicone Softener & 4 & $4-5$ & 4 & 4 & 4 & 4 & 4 \\
\hline 6 & Nano Silicone Softener & $4-5$ & $4-5$ & $4-5$ & $4-5$ & $4-5$ & 4 & $4-5$ \\
\hline
\end{tabular}

Above table indicates about the effect of different softeners like cationic, anionic, nonionic softener and silicone-based softeners on color fastness to water of enzyme treated sample. It was observed that color staining grade of enzyme wash sample with cationic, anionic, nonionic softener and silicone-based softeners is relatively same and good. The reason behind that as indigo with vat topping was used on stretch denim fabric and the water did not affect the color fastness properties. From the result, color fastness to wash of enzyme wash with cationic, anionic, nonionic softener and silicone-based softener was comparatively better.

\subsection{Effect of Different Types of Softeners on Color Fastness to Water of Stone Enzyme Treated Stretch Denim Fabric}

Table 6. Color fastness to water of stone enzyme treated stretch denim fabric.

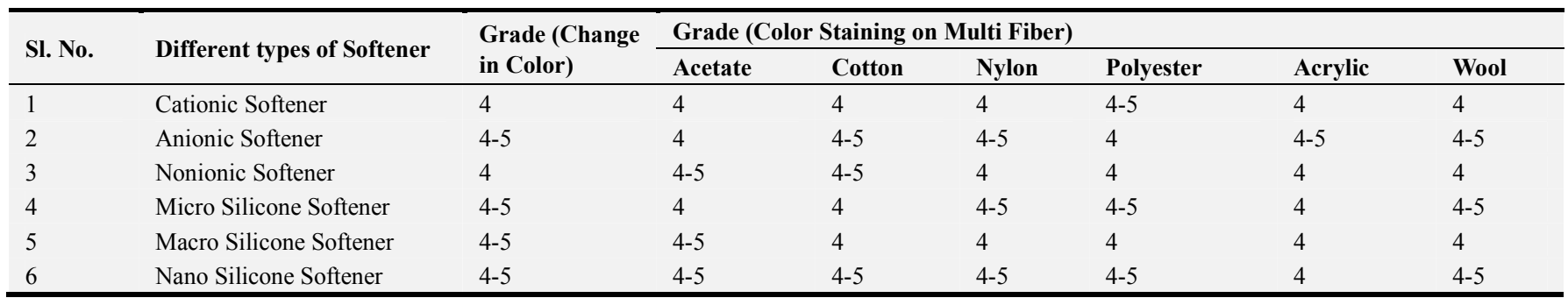

The above table characterizes about the color fastness to water of enzyme stone treated sample with cationic, anionic, nonionic softener and silicone-based softeners. It was observed that color staining result of enzyme stone wash sample with cationic, anionic, nonionic softener and siliconebased softeners was almost same and satisfactory. The reason behind that as indigo with vat topping was used on stretch denim and the water did not affect the color fastness properties. From the overall perspective, the color fastness to water for enzyme wash and stone enzyme wash treated stretch denim fabric along with different softeners was nearly similar which signified that there was no significant change at all.

\subsection{Effect of Different Softeners on Color Fastness to Light of Stretch Denim Fabric}

i) For Enzyme Wash 
Table 7. Color fastness to light of enzyme treated stretch denim fabric.

\begin{tabular}{lll}
\hline Sl. No. & Types of Softener & $\begin{array}{l}\text { Fastness Rating according to Blue } \\
\text { Wool Standard Grading }\end{array}$ \\
\hline 1 & Cationic Softener & 5 \\
2 & Anionic Softener & $4-5$ \\
3 & Nonionic Softener & $4-5$ \\
4 & Micro Silicone Softener & 5 \\
5 & Macro Silicone Softener & 5 \\
6 & Nano Silicone Softener & $5-6$ \\
\hline
\end{tabular}

The Following table shows about the effect of various softeners on color fastness to light of enzyme treated stretch denim fabric. It was observed that there was no significance outcome of softener on color fastness to light of enzyme treated stretch denim fabric. Enzyme washed with cationic, anionic and nonionic softener washed fabric was almost same grade which was very good and their degree of fade was slight. Overall, the effect of different softeners relating to color fastness to light of enzyme wash treated with cationic, anionic, nonionic and silicone-based softeners were almost same.

ii) For Stone Enzyme Wash
Table 8. Color fastness to light of stone enzyme treated stretch denim fabric.

\begin{tabular}{lll}
\hline Sl. No. & Types of Softener & $\begin{array}{l}\text { Fastness Rating according to Blue } \\
\text { Wool Standard Grading }\end{array}$ \\
\hline 1 & Cationic Softener & 5 \\
2 & Anionic Softener & 5 \\
3 & Nonionic Softener & 5 \\
4 & Micro Silicone Softener & $5-6$ \\
5 & Macro Silicone Softener & 5 \\
6 & Nano Silicone Softener & $5-6$ \\
\hline
\end{tabular}

Above table shows about the effect of softeners on color fastness to light of enzyme stone treated stretch denim fabric. No significant effect was observed for color fastness to light on stone enzyme treated stretch denim fabric. Enzyme stone wash with cationic, anionic and nonionic softeners treated fabric showed almost same grade which was good and their degree of fade was slight. So, application of different softeners on color fastness to light for both enzyme wash and enzyme stone wash treated with cationic, anionic, nonionic and silicone-based softeners displayed almost same result for stretch denim fabric.

\subsection{SEM Analysis}

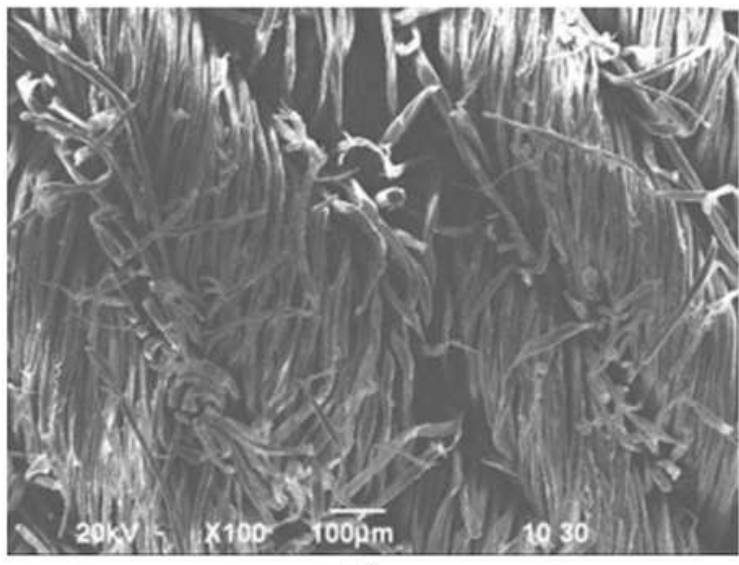

(a)

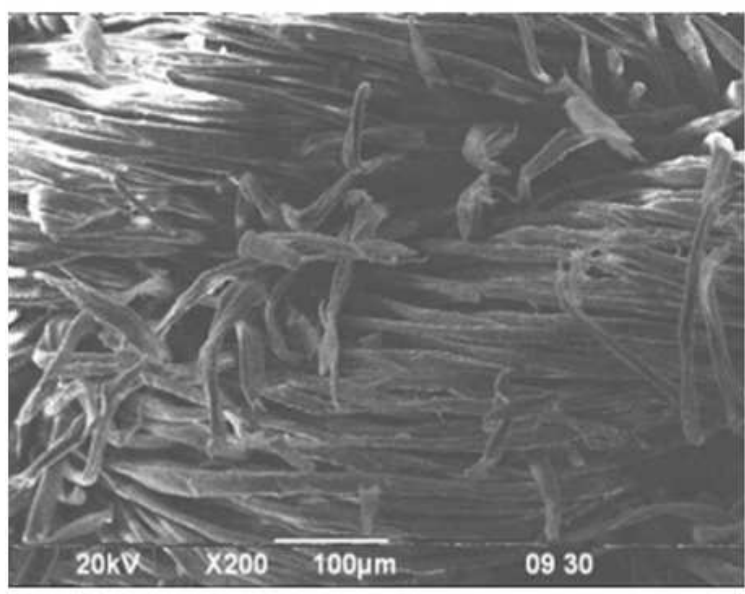

(c)

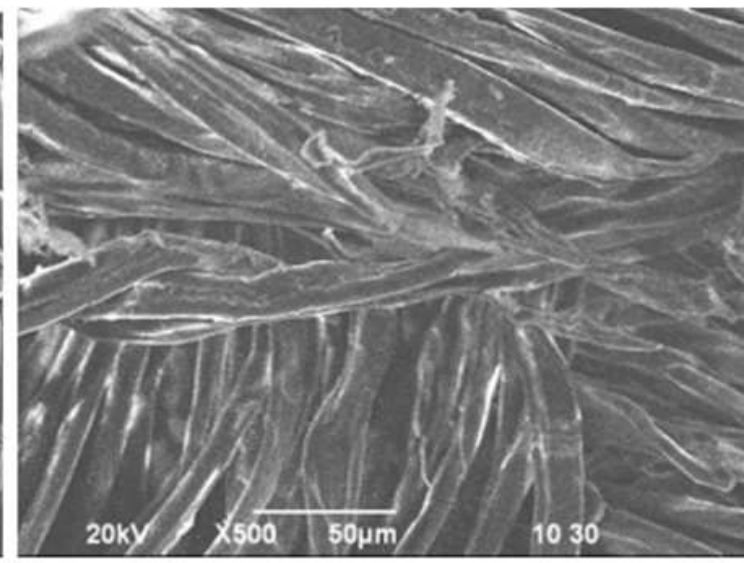

(b)

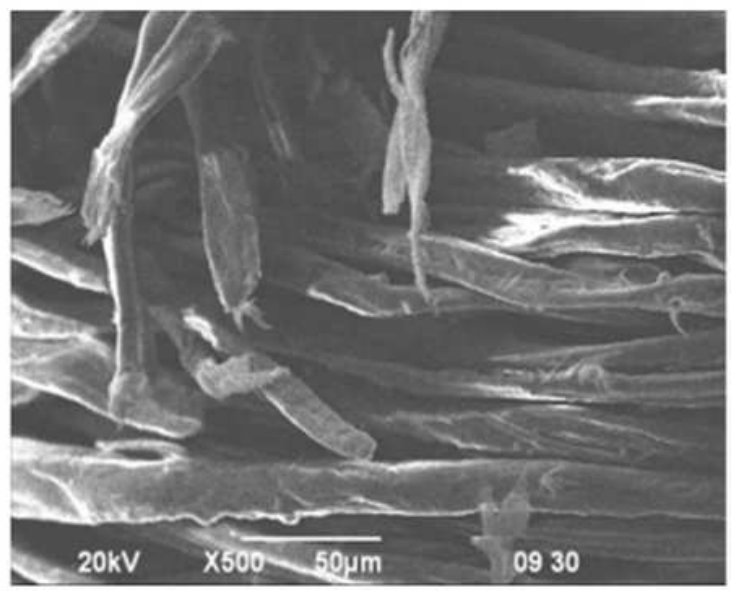

(d)

Figure 5. Scanning Electron Microscope (SEM) analysis of stretch denim fabric: (a) Cationic softener with enzyme treated stretch denim fabric (b) Micro silicone softener with enzyme treated stretch denim (c) Cationic softener with stone enzyme treated stretch denim fabric (d) Micro silicone softener with stone enzyme treated stretch denim. 
From the following figures, it was observed that enzyme wash with cationic and micro silicone softener treated stretch denim fabric implied less ruptures and less cracks than stone enzyme with cationic and micro silicone softener treated stretch denim fabric because of using pumice stones. Technically pumice stones cause wear and tear of the fabric, also creates the problem of environmental temperament of waste of the grit produced by the stones.

\section{Conclusion}

In this research work, different softeners like cationic, anionic, nonionic, silicone-based softeners were applied on stretch denim fabrics for enzyme and stone enzyme washed condition to investigate the dimensional stability and different color fastness properties. Stretch denim was selected specifically for this experiment because this type of denim fabric is considered as the most popular type right now in modern fashion world. Enzyme and enzyme stone washed sample treated with silicone-based softeners displayed better performance regarding the color fastness to wash. The grade of color change and color staining for respective color fastness to wash and color fastness to rubbing was slightly decreased for enzyme wash rather than stone enzyme wash. There was no significant effect was observed for the application of different kinds of softeners on enzyme wash and stone enzyme treated stretch denim fabric regarding the grade of color change and color staining for color fastness to light along with color fastness to water. The application of different softeners also changed the dimensional stability of stretch denim fabric. The performance was better for cationic and nonionic softeners. The GSM of stretch denim fabric also increased accordingly after applying different kinds of softeners rather than untreated stretch denim fabric. Scanning electron microscopic analysis also depicted that cationic and micro silicone softeners mostly deposited on the fibre surface. Enzyme with cationic and micro silicone softener treated stretch denim fabric implied less ruptures than stone enzyme washed with cationic and micro silicone softener treated stretch denim fabric because of using pumice stone in enzyme stone wash. Based on the findings of this research, engineers and technologists will be cautious about the use of relevant softeners for stretch denim finishing process. Such understanding will help consumers and manufacturers to achieve the best outcome from the finished products based on softeners in conjunction with dimensional stability and color fastness properties.

\section{Conflict of Interest}

All the authors do not have any possible conflicts of interest.

\section{Compliance with Ethics Requirements}

This article does not contain any studies with human or animal subjects performed by any of the authors.

\section{Acknowledgements}

The authors gratefully acknowledge the Department of Textile Engineering of Mawlana Bhashani Science and Technology University (MBSTU) and Dhaka University of Engineering and Technology (DUET) for technical support of this work.

\section{References}

[1] Khedher, F., Dhouib, S., Msahli, S., and Sakli, F. (2011). Study of the influence of matter and finishing treatments on the denim garment shade. International Journal of Clothing Science and Technology, 23 (1), 34-45.

[2] Halleb, N., Sahnoun, M., Cheikh R. M. (2019). The Influence of Finishing and Softening Treatments on The Sensory Properties of Denim Fabric. Textile and Apparel, 29 (1), 5562.

[3] Telli, A., \& Babaarslan, O. (2016). The Effect of Recycled Fibers on The Washing Performance of Denim Fabrics. The Journal of The Textile Institute, 108 (5), 812-820.

[4] Asif, A. K. M. A. \& Hasan, M. Z. (2018). Application of Nanotechnology in Modern Textiles: A Review. International Journal of Current Engineering and Technology, 8 (2), 227-231.

[5] Asif, A. K. M. A. H. (2017). An Overview of Sustainability on Apparel Manufacturing Industry in Bangladesh. Science Journal of Energy Engineering, 5 (1), 1-12.

[6] Shaheen, I. M., Mamun, M. A. A., Siddique, M. A. B., Asif, A. K. M. A. H. (2016). Effect of Finishing Machine Parameters on Dimensional Stability of Single Lacoste Cotton Knitted Fabric. Advances in Materials, 5 (5), 35-43.

[7] Eryuruk, S. H. (2019). The Effects of Elastane and Finishing Properties on Wicking, Drying and Water Vapour Permeability Properties of Denim Fabrics. International Journal of Clothing Science and Technology, 32 (2), 208-217.

[8] Roy, C. A. K. (2017). Finishing of denim fabrics. Principles of Textile Finishing, 383-415.

[9] Hasan, S. M., Asif, A. K. M. A. H., Mahmud, S. T., Shanewaz, A. K. M. (2020). An Experimental Overview of Seam Performance for Different Types of Denim Fabrics. International Journal of Current Engineering and Technology, 10 (5), 740-748.

[10] Piroi, C., Harpa, R., and Oprea, M. (2018). Regarding the Effect of Finishing Processes on Some Properties of Stretch Denim Fabrics. IOP Conference Series: Materials Science and Engineering, 459, 012059.

[11] Yildirim, N., Üstüntağ, S., and Örtlek, H. G. (2014). The Effects of Washing Treatments on Physical Properties of Denim Fabrics. Tekstil Ve Mühendis, 16-29.

[12] Çelik, H. İ., and Kaynak, H. K. (2017). An Investigation on The Effect of Elastane Draw Ratio on Air Permeability of Denim Bi-Stretch Denim Fabrics. IOP Conference Series: Materials Science and Engineering, 254 (8), 082007. 
[13] Ansari, I. Z. (2017). Impact of Stone Wash and Acid Wash on the Physical Properties of Denim. International Journal of Engineering Research, 6 (12), 499.

[14] Saleh, S. M., El-Sayed, I., and El-Shikh, A. (2012). Investigating the Impact of Enzymatic Treatment on Mechanical and Chemical Properties of Denim Fabrics. Research Journal of Textile and Apparel, 16 (2), 111-117.

[15] Siddique, M. A. B., Asif, A. K. M. A. H., Rashedul, H. K., Anwar, M. T., Saiful, I., Nusrat, N. (2016). Study on the Effect of Dyeing and Finishing Parameters on Cotton Knitted Two Thread Fleece Fabric and 1x1 Rib Fabric. Science Research, 4 (1), 7-10.

[16] Kan, C. W. (2015). Washing Techniques for Denim Jeans. Denim, 313-356.

[17] Maryan, A. S., and Montazer, M. (2014). One pot Denim Washing and Finishing Using Organo-Montmorillonite: Introducing Nano Mineral Washing and Finishing. Textile Research Journal, 85 (1), 91-100.

[18] Arikan, T., Cavusoglu, B., Alver, Y., Cil, Z. E., Akkaya, S., and Kayaoglu, B. K. (2015). Effects of Different Industrial Washing Processes on Strength and Physical Properties of Denim Fabrics. Tekstil Ve Mühendis, 22 (100), 54-68.

[19] Kan, C. W. and Yuen, C. W. M. (2012). Effect of Atmospheric Pressure Plasma Treatment on The Desizing And Subsequent Color Fading Process of Cotton Denim Fabric. Coloration Technology, 128 (5), 356-363.

[20] Park, H. R., Lee, M. C., Nishi, K. and Wakida, T. (2008). Effect of Washing and Subsequent Heat Treatment on Water
Repellency and Mechanical Properties of Nylon 6, Triacetate and Silk Fabrics Treated with Hydrocarbon Resins. Textile Coloration and Finishing, 20 (6), 87-91.

[21] Venkatraman, P. D. and Liauw, C. M. (2019). Use of A Carbon Dioxide Laser for Environmentally Beneficial Generation of Distressed/Faded Effects on Indigo Dyed Denim Fabric: Evaluation of Colour Change, Fibre Morphology, Degradation and Textile Properties. Optics and Laser Technology, 111, 701-713.

[22] Kan, C. W., Yuen, C. W. M., and Cheng, C. W. (2012). Color Fading of Indigo-Dyed Cotton Denim Fabric by Laser. Advanced Materials Research, 441, 187-191.

[23] Buscio, V., and Gutiérrez-Bouzán, C. (2017). Chemicals and Effluent Treatment in Indigo Denim Processes. Sustainability in Denim, 235-255.

[24] Sanchez, M. (2015). Dyeing of denim yarns with non-indigo dyes. Denim, 107-157.

[25] ISO 105-C10: 2006; Textiles -- Tests for color fastness--Part C04: Color fastness to washing: Test 4.

[26] ISO 105-E01: 2010; Textiles -- Tests for color fastness -- Part E01: Color fastness to water.

[27] ISO 105-X12: 2016; Textiles -- Tests for color fastness -- Part $\mathrm{X} 12$ : Color fastness to rubbing.

[28] ISO 105-B02: 2014; Textiles -- Tests for color fastness -- Part B02: Color fastness to artificial light: Xenon arc fading lamp test.

[29] ISO 23231: 2008 Textiles- Determination of dimensional change of fabrics, Accelerated machine method. 